\title{
Sistema de esgotamento sanitário e casos de diarreia em Macapá/AP
}

Macapá-AP é uma das capitais brasileiras que detém um dos piores indicadores de qualidade no sistema de esgotamento sanitário (SES). Além disso, há uma profunda escassez de estudos que tratem especificamente de correlacionar indicadores sanitários e ambientais com os de saúde pública, como o número de casos de diarreia (NOCD) nas cidades da Amazônia. Neste contexto, o objetivo da presente pesquisa foi correlacionar estatisticamente NOCD com alguns índices e sub índices de salubridade ambiental (ISA), entre outras variáveis ambientais na cidade de Macapá/Amapá/Brasil, considerando diferentes períodos sazonais. A metodologia de investigação seguiu três etapas: 1) quantificação de NOCD, categorizadas em dois períodos sazonais, o seco e o chuvoso, entre 2017 e 2018; 2) quantificação de variáveis sanitárias e ambientais disponíveis na literatura, tais como ISA, qualidade microbiológica da água de poços subterrâneos, condições sanitárias e ambientais, presença/ausência de redes de esgoto nos bairros (RE) e outras; 3) uso de sistema de informação (SIG) e análise estatística multivariada para correlacionar NOCD com as variáveis independentes. Os resultados, disponibilizados em forma de mapas espaço-sazonais e tabelas estatísticas, mostraram correlações significativas entre NOCD e as variáveis independentes, com significância para ISA (e sub índices) e RE ( $p<0,05$ ), independentemente do período sazonal. Sazonalmente, observaram-se oscilações características significativas de NOCD. Concluiu-se que, paradoxalmente, NOCD se correlaciona negativamente com ISA e RE. Portanto, rejeita-se a hipótese de que a existência de RE aumentaria ISA e reduziria NOCD em Macapá. Desta forma, percebeu-se um efeito contrário, sugerindo estatisticamente a obsolescência do SES

Palavras-chave: Saúde Pública; Fatores Sanitários e Ambientais; Variações Espaço-Sazonais; Análise Multivariada.

\section{Sewage system and cases of diarrhea in Macapá/AP}

Macapá-AP is one of the Brazilian capitals that has one of the worst quality indicators of its sewage system (SES). In addition, there is a deep scarcity of studies that specifically address correlating health and environmental indicators with those of public health, such as the number of cases of diarrhea (NOCD) in Amazonian cities. In this context, the objective of this research is statistically to correlate NOCD with some indices and sub-indices of environmental health (ISA), among other environmental variables in the city of Macapá/Amapá/Brazil considering two different seasonal periods. The research methodology followed three stages: 1) quantification of NOCD, categorized into two seasonal periods, dry and rainy, between 2017 and 2018; 2) quantification of sanitary and environmental variables available in the literature, such as ISA, microbiological quality of water from underground wells, sanitary and environmental conditions, presence/absence of sewage networks in neighborhoods (RE), etc.; 3) use of geographic information system (GIS) and multivariate statistical analysis to correlate NOCD with independent variables. The results, made available in the form of space-seasonal maps and statistical tables, show significant correlations between NOCD and independent variables, with significance for ISA (and sub-indices) and RE $(p<0.05)$, regardless of seasonal period. Seasonally, significant characteristic oscillations of NOCD were observed. We conclude, paradoxically, that NOCD correlates negatively with ISA and RE. Therefore, the hypothesis is rejected that where there is RE or better ISA in Macapá should impact on the reduction of NOCD. But when statistically tested, an opposite effect was observed in NOCD.

Keywords: Public Health; Sanitary and Environmental Factors; Space-seasonal Variations; Multivariate Analysis.

\section{Topic: Engenharia Sanitária}

Reviewed anonymously in the process of blind peer.
Received: 04/02/2021

Approved: 24/02/2021
Clezio Junior Teixeira Viegas (iD)

Universidade Federal do Amapá, Brasil

http://lattes.cnpq.br/8475916901983488

http://orcid.org/0000-0002-1558-9052

irclezio.2017@gmail.com

\section{Taís Silva Sousa}

Universidade Federal do Amapá, Brasil http://lattes.cnpq.br/2341265184565372 http://orcid.org/0000-0003-3258-4621 taisousa155@gmail.com

Helenilza Ferreira Albuquerque Cunha Universidade Federal do Amapá, Brasil http://lattes.cnpq.br/3443290397582063 http://orcid.org/0000-0001-7101-9305

helenilzacunha@gmail.com

\author{
Alan Cavalcanti da Cunha (iD \\ Universidade Federal do Amapá, Brasil \\ http://lattes.cnpq.br/2181817533284030 \\ http://orcid.org/0000-0002-1846-9486 \\ alancunha12@gmail.com
}

Referencing this:

VIEGAS, C. J. T.; SOUSA, T. S.; CUNHA, H. F. A.; CUNHA, A. C.. Sistema de esgotamento sanitário e casos de diarreia em Macapá/AP. Revista Ibero Americana de Ciências Ambientais, v.12, n.2, p.303-316, 2021. DOI: http://doi.org/10.6008/CBPC2179-6858.2021.002.0028 


\section{INTRODUÇÃO}

O saneamento básico ainda é um desafio global, pois cerca de 2,3 bilhões de pessoas não possuem acesso a serviços sanitários. No Brasil, a situação também é complexa. Entre 194 países no ranking da OMS, o país está na 103a colocação em saneamento (OMS et al., 2017; OMS, 2017).

Na região Norte o saneamento é desafiador, prevalecendo os piores índices do país, e a cidade de Macapá é uma das mais críticas. No ranking do saneamento básico do Sistema Nacional de Informação Sobre Saneamento (SNIS), dentre as 100 maiores cidades brasileiras, Macapá ocupa a 99a colocação, e isto provavelmente pode ser um reflexo da menor participação do país em investimentos no setor, variando de R\$ 0 a 5 milhões entre os anos de 2016 a 2018 (INSTITUTO TRATA BRASIL, 2020; BRASIL, 2019; SNIS, 2019).

Essa deficiência ou até mesmo ausência de SES tem causado complicações de saúde pública, devido à contaminação dos corpos d'água por esgoto doméstico, e este é um dos fatores responsáveis pela disseminação de doenças causadas por vírus, bactérias e protozoários. Devido ao contato da população com estas águas, estes fatores têm sido potencializados, servindo como fontes de contaminação e degradação da qualidade da água de corpos receptores, e isto têm ocorrido em muitas cidades costeiras da Amazônia (ANNE-SOPHIE et al., 2015; GROTT et al., 2018; PEREIRA et al., 2019; ABREU et al., 2020).

Alguns exemplos destes impactos negativos, diretos ou indiretos, incidem sobre a força laboral e a economia, com redução dos múltiplos usos das águas, principalmente do setor de abastecimento público (OLIVEIRA et al., 2019), turismo, saúde e ecossistemas aquáticos (ARAUJO et al., 2019). Nestas circunstâncias, o esgoto doméstico tem sido integrado com a rede de drenagem que percola o solo até atingir as águas subterrâneas desprotegidas. E, como não há praticamente nenhum tratamento adequado do esgoto doméstico, este tem sido despejado in natura nos corpos d'água, prejudicando mananciais com impactos na captação de água da concessionária no rio Amazonas (GROTT et al., 2018; CUNHA et al., 2014).

Em face das potenciais e desconhecidas consequências da precariedade do SES em Macapá, associadas com indicadores de saúde pública, especialmente as notificações de casos de diarreia (NOCD), a pesquisa testou a seguinte hipótese: os bairros medianamente salubres (ISA mediano), em contraste com os de baixa salubridade (ISA baixo) (SANTOS, 2012), apresentam menor número de notificações de casos de diarreia (NOCD), independente do período sazonal, além da presença/ausência da RE e da localidade dos bairros.

Portanto, a pesquisa objetivou testar estatisticamente se houve influência da infraestrutura e dos serviços de esgotamento sanitário disponíveis (RE) sobre NODC; correlacionar NOCD com variáveis sanitárias e ambientais (ISA e seus sub índices nos bairros), qualidade da água de poços subterrâneos (GROTT et al., 2018), presença/ausência de RE, e características ambientais dos bairros urbanos; testar estatisticamente as variações espaço-sazonais de NOCD e utilizar mapas de calor (espaço-sazonais) para identificar quando e quais bairros apresentaram maiores NODC, a fim de interpretar como as variáveis sanitárias e ambientais explicam tais variações. 


\section{MEATERIAIS E MÉTODOS}

\section{Área de estudo}

O município de Macapá situa-se no sudeste do estado do Amapá, na Amazônia setentrional, região norte do Brasil. A área urbana compreende a porção sul do município, entre as coordenadas $02^{\prime} 18.84^{\prime \prime} \mathrm{N}$; 513'59.1"W; limitando-se a oeste ao município de Santana e a leste pelo rio Amazonas. Possui acesso por via aérea e fluvial, não havendo interligação terrestre com outras capitais do Brasil. É a única capital brasileira cortada pela linha do equador e localizada às margens do rio Amazonas, com população estimada em 503.327 pessoas (IBGE, 2019). Seu sistema de drenagem está inserido nas bacias do Igarapé da Fortaleza ( $\approx 95 \%$ da área urbana) e do Curiaú ( $\approx 5 \%)$. O foco da pesquisa foi a bacia do Igarapé da Fortaleza, que faz limite entre as sedes de Macapá, Santana e o rio Amazonas (AGUIAR et al., 2003; ABREU et al., 2020).

O ISA dos bairros de Macapá foi estimado por Santos (2012), os quais supõe-se não sofrer alterações significativas de infraestruturas nos últimos 10 anos. O ISA dos bairros de Macapá varia somente entre baixa e média salubridade. As áreas mais salubres são as mais próximas ao centro, onde há melhor infraestrutura sanitária (SANTOS, 2012) (Figura 1).

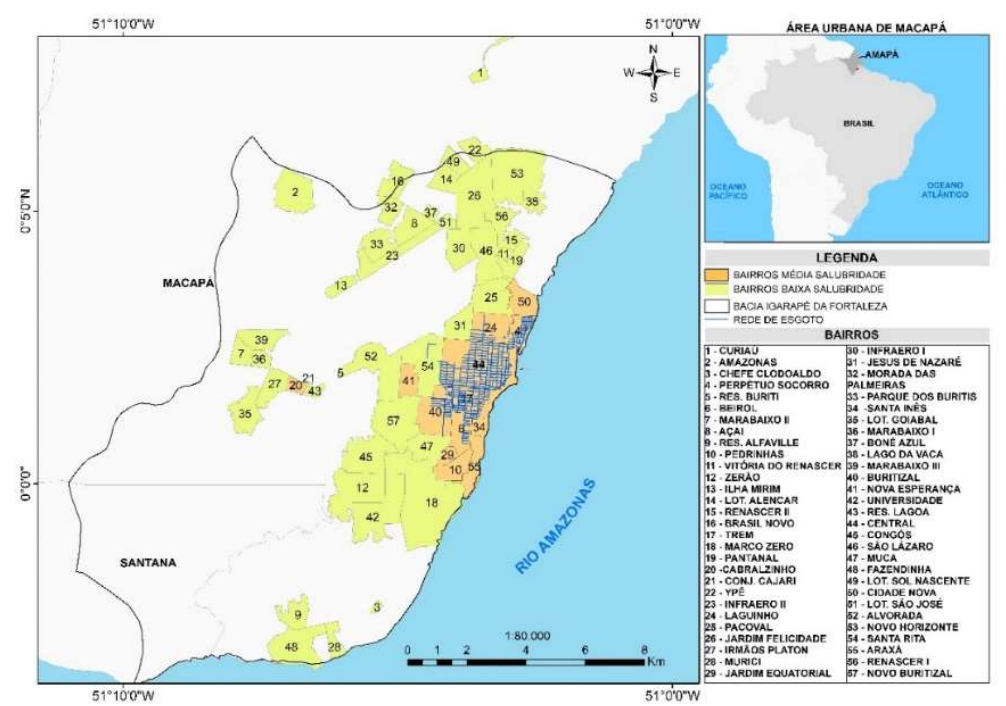

Figura 1: Zona urbana de Macapá: bairros, bacia do Igarapé da Fortaleza, Salubridade Ambiental (ISA) e Rede de Esgoto (RE). Fonte: adaptado de Santos (2012) e Base de Dados da Secretaria de Estado do Meio Ambiente SEMA/AP.

\section{Uso de SIG para elaboração de mapas de variações espaço-sazonais de NOCD}

\section{Infraestrutura da Rede de Esgoto Sanitário (SES)}

Os Mapas da infraestrutura da rede de esgoto foram gerados a partir da base de dados Planialtimétricos do ano de 2016 da Companhia de Água e Esgoto de Macapá - CAESA. Esta base foi gentilmente disponibilizada pela Secretaria de Estado do Meio Ambiente - SEMA/AP, em formato DWG (AutoCad). O tratamento destes dados consistiu na conversão do formato disponibilizado para SHP (Shapefile), utilizando o Software ArcMap, versão 10.5. Este procedimento permitiu a utilização das informações sobre a rede de esgoto (RE) e a infraestrutura do SES na elaboração de mapas com a ferramenta ArcGis. 


\section{Número de ocorrência de casos de diarreia NOCD}

Os dados de NOCD entre os anos de 2017 e 2018 foram disponibilizados pela Secretaria Municipal

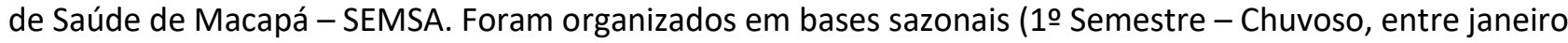
a junho, e 2o Semestre - Menos Chuvoso ou Seco, entre julho a dezembro) e classificados por bairro (GROTT et al., 2018; TAVARES, 2015).

\section{Variáveis sanitárias e ambientais}

As variáveis sanitárias e ambientais utilizadas foram: ISA e sub-índices (lab - Índice de abastecimento de Água, les - Índice de Esgoto Sanitário, Idr - Índice de Drenagem e Irs - Índice de Atendimento de Resíduos Sólidos), disponíveis para o ano de 2012 (SANTOS, 2012); qualidade da água subterrânea de alguns bairros de Macapá, coletadas em diferentes períodos sazonais, e disponíveis para os anos de 2014 e 2015 (GROTT et al., 2018). Os dados do ISA foram utilizados como variável independente (baixa ou média salubridade) para explicar as variações de NOCD. Para integrá-las com a série de NOCD, mais atuais, considerou-se que ambas não se alteraram significativamente durante este lapso temporal.

\section{Integração estatística entre NOCD e variáveis sanitárias e ambientais}

A espacialização das informações de NOCD foi realizada em função da existência/ausência de infraestrutura da rede de esgoto sanitário (RE) por bairros, identificado com presença (1) e ausência (0); classificação do ISA (baixa ou média salubridade) e características ambientais (qualidade da água subterrânea, tipologia da infraestrutura das residências, proximidades com corpos d'água - rede de drenagem), no contexto da bacia hidrográfica do Igarapé da Fortaleza.

Subsequentemente foi comparada a evolução espaço-sazonal de NCOD, de acordo com a evolução dessas notificações por bairro da zona urbana de Macapá. Uma matriz de dados com todas as variáveis e fatores foi gerada e analisada para testar as hipóteses $(p<0,05)$.

Do ponto de vista do GIS os dados foram integrados em forma de mapas de calor representando as variações espaço-sazonais de NOCD, sua frequência e distribuição por bairro e por período sazonal. Como os mapas de calor gerados são apenas demonstrativos da variação espaço-sazonal do NOCD, a tabela 2 foi construída para resumir e complementar todas as correlações significativas entre as variáveis independentes e NOCD em Macapá.

\section{Análise estatística}

Foi utilizado um banco de dados com múltiplas variáveis de qualidade da água (GROTT et al., 2018) e ISA (SANTOS, 2012), ordenadas de acordo com a tabela 1, em um arquivo em formato ".txt" exclusivamente analisadas no software R (R TEAM, 2018). Os parâmetros de qualidade da água e os NOCD foram classificados de acordo com o período sazonal (1ㅇs semestre - chuvoso, e 2o semestre - seco). Os parâmetros sanitários e ambientais e a RE foram classificados pela presença (1), ausência (0), bem como variáveis de identificação 
sítios amostrais (Tabela 1).

A análise estatística permitiu testar diferentes respostas de NOCD (variável dependente) em função das demais variáveis independentes. Foram utilizados os testes estatísticos de Wilcoxon pareado e Friedman (R CORE TEAM, 2018), para comparar NOCD entre dois ou mais períodos (chuvoso e o seco de 2017 e 2018). O método de Friedman foi necessário para testar hipóteses de até quatro comparações simultâneas. Uma análise multivariada de covariância (ANCOVA) (CRAWLEY, 2007) foi utilizada para testar os melhores modelos propostos das análises de regressões, ranqueando-os em função da melhor explicabilidade das variáveis $\left(R_{\mathrm{aj}}{ }^{2}\right)$ e fatores sanitários e ambientais avaliados (GROTT et al., 2018) em relação aos respectivos NOCD.

Como a distribuição de frequências das variáveis foram, em sua maioria, não normais, foram utilizadas as medianas como parâmetros de medidas de tendência central. Assim, foi possível comparar o NOCD nos períodos seco e chuvoso de 2017 e 2018. Na comparação dos quatro semestres simultâneos, observou-se um comportamento sazonal oscilatório das medianas, com picos de NOCD nos períodos chuvosos e mínimos nos períodos secos. Este comportamento foi estatisticamente avaliado, utilizando-se o método de Friedman (CRAWLEY, 2007).

Tabela 1: Variáveis utilizadas na matriz de correlação estatística

\begin{tabular}{|c|c|c|c|c|}
\hline \multirow{2}{*}{\multicolumn{2}{|c|}{ Parâmetro }} & \multicolumn{2}{|l|}{ Média } & \multirow{2}{*}{ Explicação } \\
\hline & & Chuvoso (c) & $\operatorname{Seco}(\mathrm{s})$ & \\
\hline \multirow{6}{*}{ 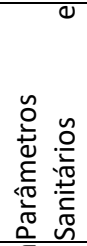 } & CodPoco & $\begin{array}{l}---- \\
--\end{array}$ & & Código do Poço (Identificação) \\
\hline & Bairro & ----- & & № do Bairro \\
\hline & Lat & ----- & & Latitude \\
\hline & Long & ----- & & Longitude \\
\hline & Caixa_D'água & ----- & & Presença/Ausência (Reservatório) \\
\hline & Filtro & ----- & & Presença/Ausência (Filtro) \\
\hline \multirow{11}{*}{ 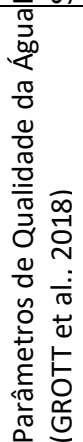 } & $\mathrm{pH}$ & 4,72 & 4,36 & Acidez (sazonal) \\
\hline & Turb & 2,82 & 1,95 & Turbidez (sazonal) \\
\hline & Cor & 34,98 & 24,73 & Cor (sazonal) \\
\hline & $\mathrm{Fe}$ & 0,11 & NA & Concentração de Ferro (sazonal) \\
\hline & $\mathrm{NO}_{3}$ & 2,22 & 2,61 & Concentração de Nitrato (sazonal) \\
\hline & $\mathrm{Mn}$ & 0,02 & 0,08 & Concentração de Manganês (sazonal) \\
\hline & $\mathrm{Cl}$ & 32,59 & 14,74 & Concentração de Cloreto (sazonal) \\
\hline & $\mathrm{NH}_{3}$ & 0,60 & 0,63 & Concentração de Amônia (sazonal) \\
\hline & & 44,34 & 21,48 & Concentração de Alumínio (sazonal) \\
\hline & Colif. Total & 576,60 & 75,91 & Coliforme Total/100 mL (sazonal) \\
\hline & E.coli & 29,87 & 4,88 & E.coli/100 mL (sazonal) \\
\hline \multirow{5}{*}{ 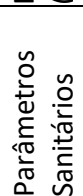 } & ISA (Salub) & 0,47 & & Índice de Salubridade Ambiental \\
\hline & lab & 0,50 & & Índice de Abastecimento de Água \\
\hline & les & 0,32 & & Índice de Esgotamento Sanitário \\
\hline & Idu & 0,48 & & Índice de Drenagem (cobertura) \\
\hline & & 0,79 & & Índice de Atendimento. Res. Sol. \\
\hline \multirow{4}{*}{ 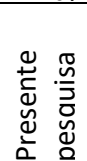 } & $\mathrm{RE}$ & $1 / 0$ & & Rede de Esgoto (Presença / Ausência) \\
\hline & Dens & 81,645 & & Densidade Populacional/Bairro \\
\hline & Diar1Sem2017 / Diar2Sem2017 & 310,96 & 205,37 & NOCD 1 e $2 \circ$ semestres 2017 (Sazonal) \\
\hline & Diar1Sem2018 / Diar2Sem2018 & 361,69 & 235,98 & NOCD 1ㅇ e 2ㅇ semestres 2018 (Sazonal) \\
\hline
\end{tabular}

\section{RESULTADOS}

\section{NOCD e a variação sazonal de 2017 e 2018}

Testes estatísticos de Friedman e Wilcoxon $(p<0,05)$ indicaram diferenças significativas entre todas as medianas semestrais de NOCD de 2017 e 2018 (Figura 4) ( $<<0,01$ ). Quando os períodos do mesmo ano 
são testados por regressão linear, a correlação apresenta coeficiente de determinação $R^{2}{ }_{\text {aj }}=0,96$, com $\mathrm{p}<0.01, \mathrm{~F}_{(47,95 \%)}=1232$ (muito significativo).

De modo similar, avaliando só os 20 semestres (2017 e 2018), a regressão linear resultou em $\mathrm{R}^{2}{ }_{\text {aj }}=$ 0,91 , com $p<0.01$ e $\mathrm{F}_{(49,95 \%)}=507,4$ (muito significativo). Isso sugere que o número dos casos de diarreia no 20 semestre de 2017 (seco) explica em aproximadamente 91\% a variação das ocorrências no período seco do 2 을 semestre de 2018 ('padrão').

Portanto, em ambos os períodos há correlação entre os NOCD de períodos anteriores, sugerindo uma tendência de crescimento interanual e dependente do NOCD do semestre ou do ano anterior. Por exemplo, o teste estatístico pareado de Wilcoxon (R CORE TEAM, 2018) indicou também diferenças significativas, com $p<0,001, G L=51, V=4851$, para o 1o semestre dos respectivos anos. Do mesmo modo, o teste de Wilcoxon resultou em $p<0,001, G L=51, V=5050$ (significativo) para o 2올 semestre. Em ambos os períodos, a tendência temporal é sempre de crescimento oscilante do NOCD entre 2017 e 2018.

\section{Comparações entre períodos do 10 e 20 semestres do mesmo ano (2017 ou 2018)}

Os resultados das análises estatísticas indicaram significativa e decrescente variação de NOCD em 2017 (medianas dos números de casos do 1ํe e 2o semestres). O teste pareado de Wilcoxon (R CORE TEAM, 2018 ) indicou $p<0,001, G L=51, V=5050$ (muito significativo). Do mesmo modo, para os mesmos períodos de 2018, o teste de Wilcoxon resultou em $p<0,001, G L=51, V=4851$ (muito significativo). Comparando-se os NOCD do mesmo ano, há diferenças estatísticas significativas entre ambos os períodos, porém menor no segundo semestre, isto é, no período seco (Figura 4).

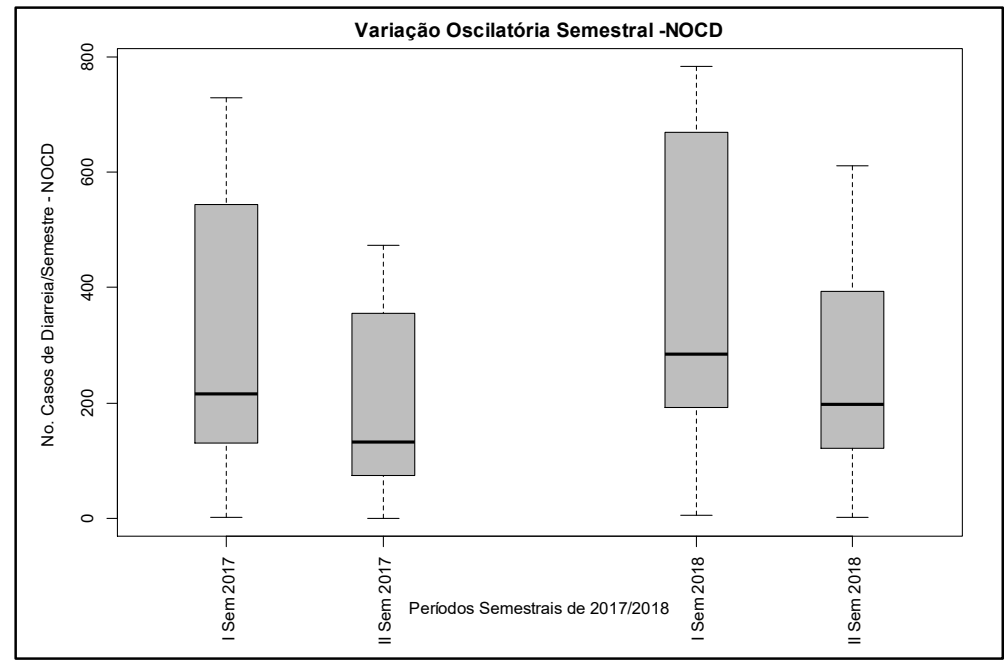

Figura 4: Variação com padrão oscilatório do número de casos de diarreia (NOCD) comparando intra-anual para os 10 e 20 semestres 2017 e 2018.

Ao se testar as tendências da evolução das medianas e variações das ocorrências de diarreia entre diferentes anos (2017 e 2018), confirmou-se o padrão de redução do número de casos de diarreia entre o 1응 e o 2 ㅇ semestres. Portanto, durante os 2ํㅗ semestres de 2017 e 2018, ocorreu redução significativa em relação às ocorrências dos 1으 semestres dos respectivos anos. A regressão linear para avaliar a evolução intra-anual resultou em $R^{2}{ }_{a j}=0,97$ e $p<0.01$ e $F_{(47,95 \%)}=1793$ (muito significativo). 
Analisando todos os quatro semestres conjuntamente, observou-se um padrão de oscilação das medianas de NOCD (redução intra-anual e crescimento interanual), com maiores picos e variâncias nos períodos chuvosos, invertendo-se a tendência nos períodos secos (Figura 4). Isso indica uma oscilante variação de NOCD no tempo e no espaço, confirmando-se a hipótese de significativa variação para qualquer combinação comparativa entre as quatro medianas. Foi utilizado o método de Friedman (R CORE TEAM, 2018) $\left(p<0,001, G L=3, \chi^{2}=111,36\right.$, muito significativo $)$.

\section{Relação entre parâmetros sanitários e ambientais e NOCD}

Uma análise preliminar multivariada (ANCOVA) foi realizada em relação aos casos de diarreia entre 2017 e 2018, em função das seguintes variáveis independentes descritas a seguir: a presença ou ausência da RE resultou em alta significância sobre o NOCD. Somente este parâmetro explica em média 50\% da variação total de NOCD no período entre 2017 e 2018 (Tabela 2). Isto é, NOCD é positiva e significativamente influenciado pela presença da RE. Porém, está correlação deveria ser inversa, com sinal negativo, significando que a sua existência não significa que o SES esteja funcionando ou operando eficientemente. Para esta análise, o intervalo da variação do coeficiente de determinação entre NOCD e RE variou entre $0,51 \leq R^{2}{ }_{\mathrm{aj}} \leq 0,56$, e $p<0,05$ (Figura 5).

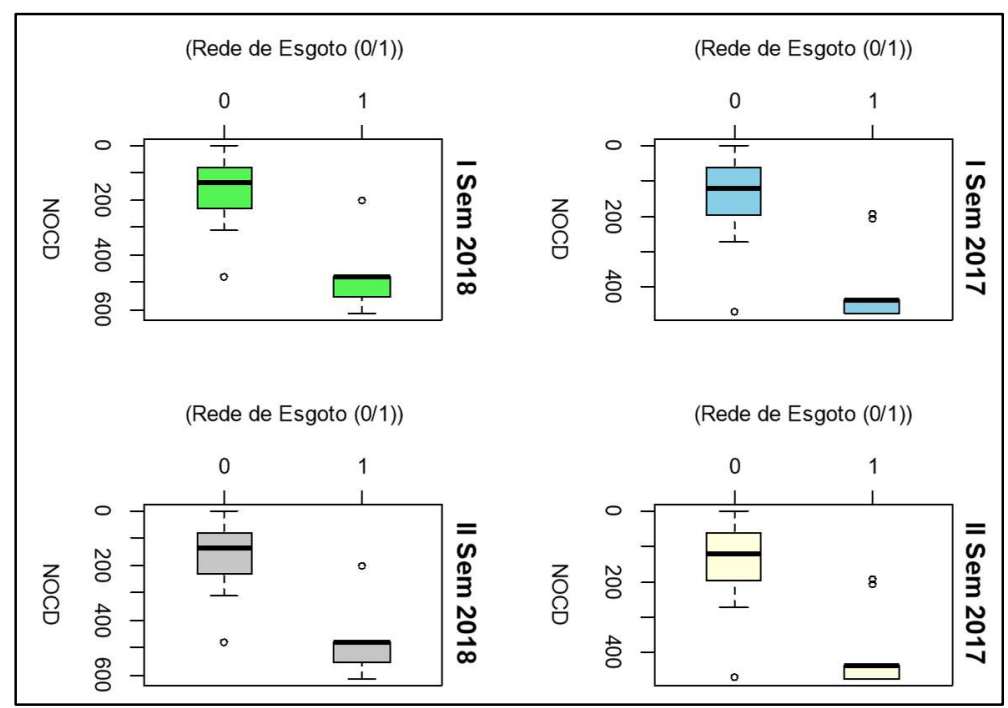

Figura 5: Análise de Box Plot Agrupada com variações de casos de diarreia segundo o fator RE ou Salub entre 2017 e 2018 (0 = ausência de RE e 1 = presença de RE). Fonte: adaptado de Grott et al. (2018); CAESA (2016) e SEMSA.

Por outro lado, nenhum parâmetro de qualidade de água teve influência sobre NOCD, sem resultado significativo. Esses parâmetros, isoladamente em cada regressão, influenciaram apenas de 1 a $9 \%\left(R_{\mathrm{aj}}{ }^{2}\right)$. Para representar está correlação na análise final, foram incluídos apenas CT e E.coli (em tese, relacionados com NOCD).

Com este resultado, retorna-se ao paradoxo do SES da cidade de Macapá. Infere-se que, onde o SES é teoricamente mais estruturado (presença da RE e ISA mediano), é justamente onde há significância e correlação com o aumento de NOCD. Isto é, os equipamentos que compõem todo o SES existente estão muito concentrados nas zonas mais populosas da cidade, refletindo-se indubitavelmente nas zonas ou 
bairros com cores mais 'quentes' da malha urbana de Macapá e coincidentes com esta antiga infraestrutura de esgoto sanitário indicados nos mapas (bairros 40, 44 e 54 das Figuras 2 e 3).

Tabela 2: ARM que explicam o NOCD $\left(Y_{i}\right)$ em relação às variáveis sanitárias, ambientais e demográficas (Grott et al., 2018; Santos, 2012 e presente pesquisa). Células hachuriadas em cinza indicam significância (*)

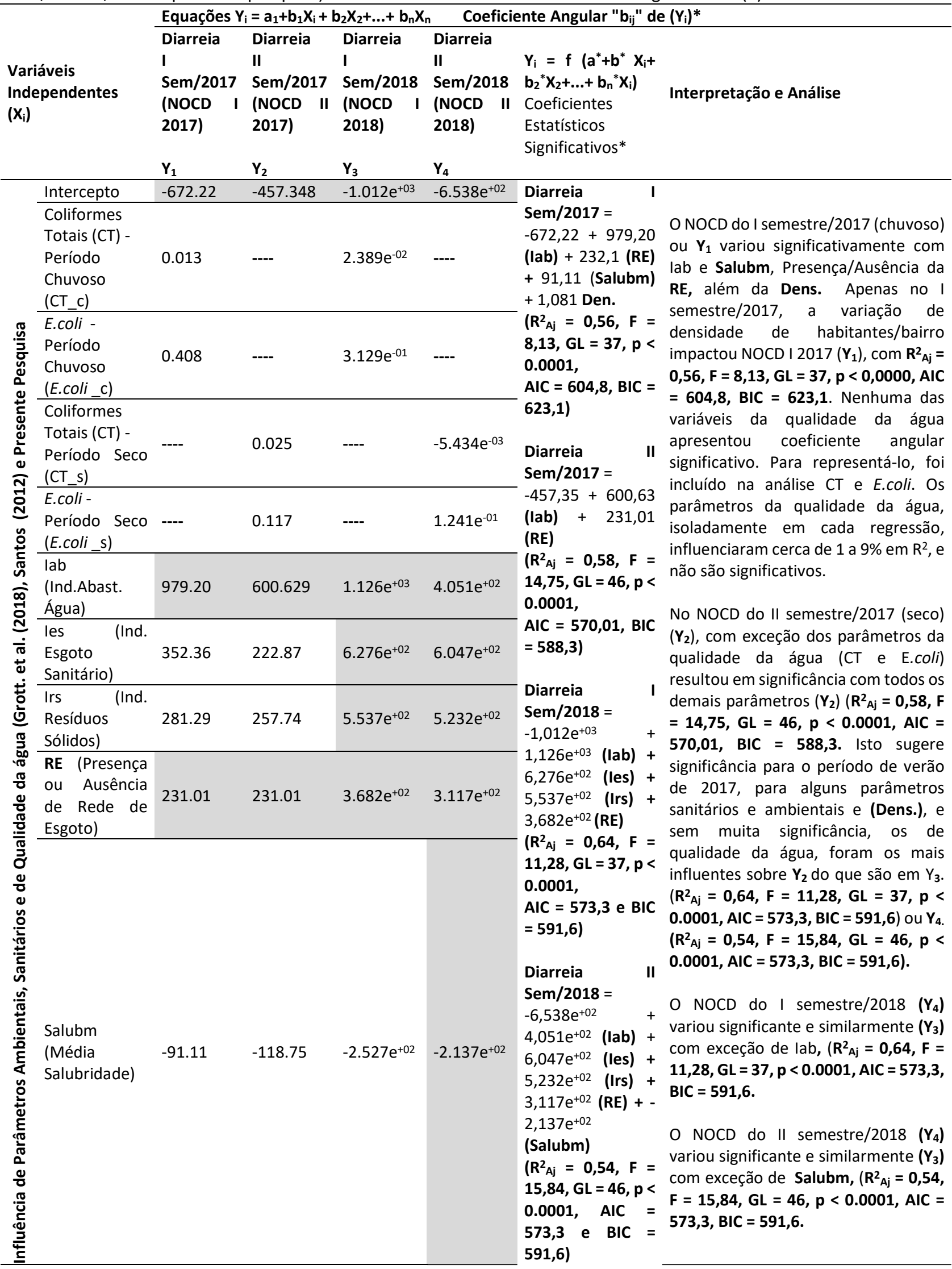




Densidade
$\begin{aligned} & \text { Populacional } \\ & \text { do } 1.084\end{aligned}$
$\begin{aligned} & \text { (Dens.) } \\ & \text { (Dairro }\end{aligned}$

Nas equações de regressões foram consideradas: colunas em cor cinza são os coeficientes significativos, em cor branca são não significativos.

Salubm = índice de salubridade média ou moderada. Salubb = o índice de salubridade baixa, não foi significativo nas análises.

AIC (Parâmetro Estatístico Akaike) e BIC (Parâmetro Estatístico Bayesiano) utilizados para a escolha do modelo mais parcimonioso da análise (melhor $\mathrm{R}^{2}$ combinado com o menor número de parâmetros do modelo).

\section{DISCUSSÃO}

Nas figuras 2 e 3 observa-se que os bairros com maior NOCD estão mais próximos à rede de esgoto da cidade (RE), isto é, bairros considerados medianamente salubres (ISA médios), indicados por cores mais quentes (vermelhas). Nestes casos, é possível observar que estão localizados nas regiões mais baixas da cidade, próximas do rio Amazonas e circundados por corpos d'água conhecidos como "áreas de ressaca", sujeitos às variações de marés e maior risco de alagamentos (SOUSA et al., 2019).

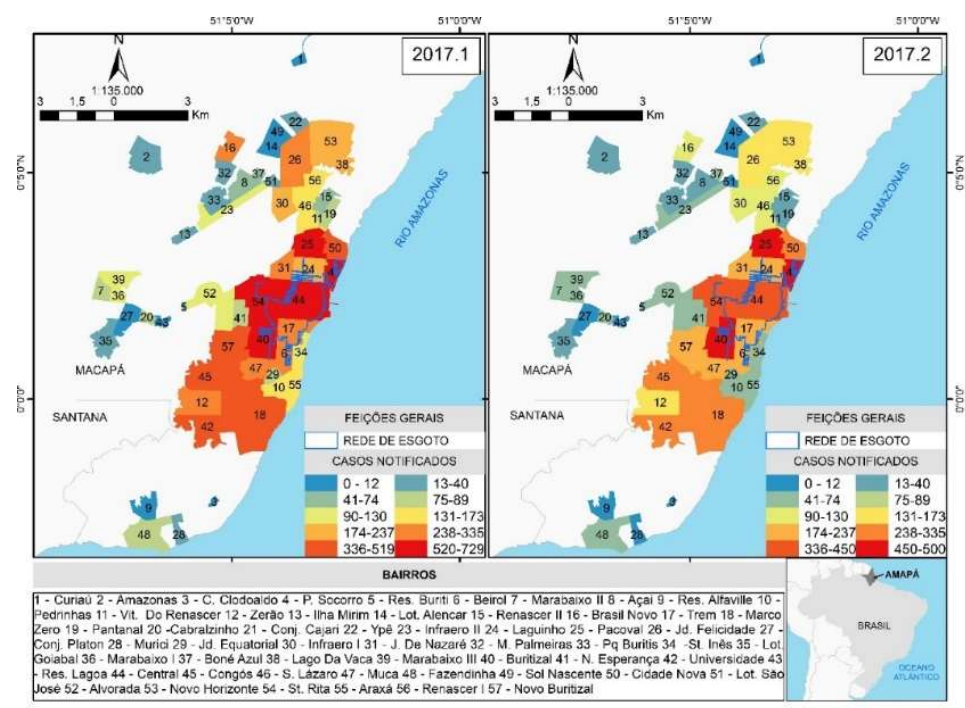

Figura 2: Casos notificados de diarreia por bairro em cada semestre do ano de 2017. Fonte: adaptado da base de dados da SEMA e da SEMSA.

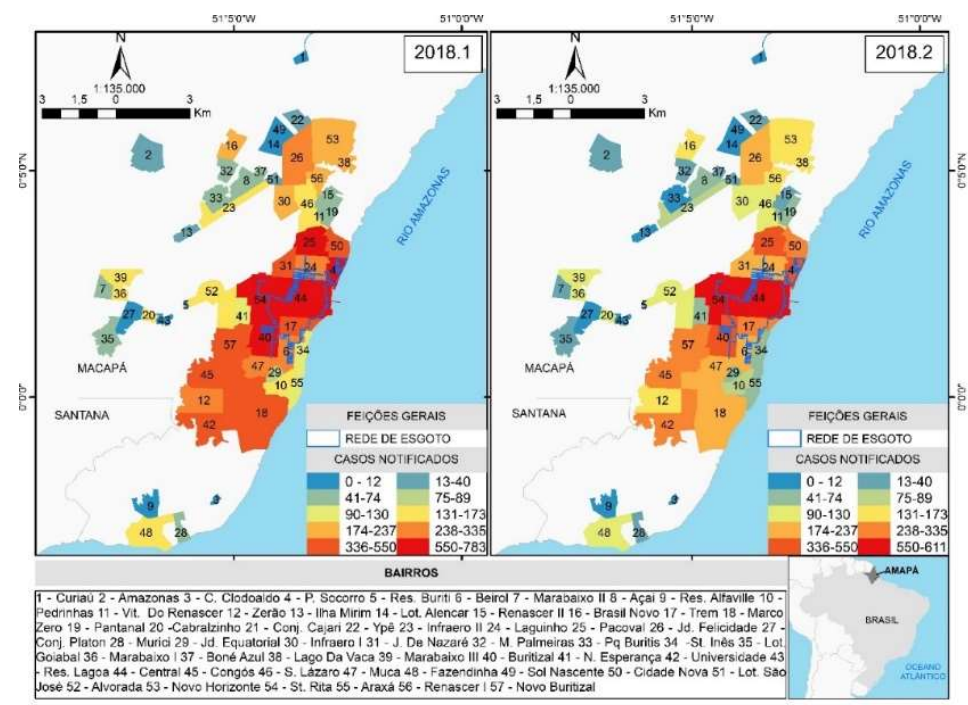

Figura 3: Casos notificados de diarreia por bairro em cada semestre do ano de 2018. Fonte adaptado da base de dados da SEMA e da SEMSA. 
A área com RE, representada pelo polígono azul das figuras 2 e 3, é relativamente pequena comparada com toda a área urbana. Por exemplo, a RE ocupa uma área de apenas $4,33 \mathrm{~km}^{2}$ dos $128,37 \mathrm{~km}^{2}$ da mancha urbana da cidade, ou seja, 3,37\%, um valor muito baixo, mesmo para os padrões amazônicos (VIEGAS et al., 2019).

Observa-se que o NOCD é tanto mais intenso nos períodos chuvosos em comparação com os períodos secos quanto nas áreas onde há a presença de RE (cores mais quentes). Ou seja, um paradoxo do ponto de vista sanitário, onde o perímetro mais saneado (ISA de média salubridade) (SANTOS, 2012), o NOCD é mais frequente e intenso. Historicamente o SES da cidade é deficiente, e a oferta dos serviços de saneamento não tem sido proporcional à expansão demográfica da cidade (CHAGAS et al., 2016).

No cenário projetado de um SES em 1974, recebendo poucas melhorias e com baixa taxa de investimento (0,002\% a.a.) em relação ao restante do país, há apenas uma fração do esgoto tratado por uma lagoa de estabilização biológica colapsada (MACAPÁ, 2016; BRASIL, 2019). Portanto, o SES parece não refletir qualquer redução de NOCD no tempo.

Como a oferta de SES é muito baixa, atendendo apenas 54.994 habitantes, ou 10,91\% da população estimada (BRASIL, 2019; IBGE, 2019; SNIS, 2019), é comum que a população faça uso de soluções individuais, como fossas construídas sem rigor técnico e, quando possível, os resíduos são recolhidos e eventualmente transportados por caminhões "limpa fossa" para uma única lagoa de estabilização (CUNHA et al., 2004; CUNHA et al., 2005; GROTT et al., 2018).

Como visto anteriormente existe um padrão oscilatório do NOCD em Macapá. Isso sugere que as condições sanitárias estão intima e sazonalmente mais correlacionados com maior precipitação, onde se percebe maior influência sobre NOCD. Como resultado, é possível admitir que a população em geral está mais exposta ao contato direto com águas pluviais contaminadas por esgotos domésticos ou extravasados do SES. E, portanto, nestes cenários há maior probabilidade de que os agentes etiológicos causadores da diarreia atuem com mais eficiência (MACAPÁ, 2016; GROTT et al., 2018, VIEGAS et al., 2019; SOUSA et al., 2019; OLIVEIRA et al., 2014).

Além disso, algumas explicações sobre a tendência de aumento de NOCD no tempo decorrem do fato das deficiências do SES (média e baixa salubridade) não dispor de sistemas de separação absoluta entre águas pluviais e esgoto doméstico, o que parece acentuar ainda mais o NOCD. Esse fato tem sido observado em estudos similares da literatura, corroborando esta tendência (HELLER et al., 2003; IMADA et al., 2016; BIDKHORI et al., 2018; FILE et al., 2019; MIAO et al., 2019).

Desta forma, os SES dos centros urbanos de grandes e médias cidades têm se tornado cada vez mais ineficientes, obsoletos ou inexistentes, sem a capacidade de se manter ou se expandir para atender a demanda de serviços com a crescente taxa de expansão urbana, especialmente pela falta planejamento e de investimentos (DECINA et al., 2016).

Os casos de diarreia, frequentemente apresentam correlação com qualidade da água (HELLER et al., 2003; CASTRO et al., 2009; IMADA et al., 2016; VAN BIJNEN et al., 2018; VIEGAS et al., 2019; SOUSA et al., 2019; OLIVEIRA et al., 2019). Mas, no presente caso, quando as variáveis de consumo de água subterrânea 
(GROTT et al., 2018) são inseridas num modelo de regressão múltipla, o resultado é não significativo para qualquer um dos parâmetros. Por exemplo, os testes estatísticos mostraram não haver correlação entre o consumo das águas dos poços analisados por Grott et al. (2018) e NOCD. Contudo, é importante frisar que os referidos autores coletaram amostras de água apenas entre 2014 e 2015, ocorrendo um lapso temporal entre as medidas de qualidade da água analisadas e o período mais atual do registro das ocorrências das doenças (NOCD). Neste caso, este fato reforça o argumento de que a variação de NOCD está relacionada com outros fatores, como drenagem, dinâmica das águas superficiais, águas pluviais (Idr) e a capacidade de autodepuração dos mananciais (lab). Estes, no entanto, são parâmetros pouco conhecidos e estudados localmente, mas variantes sazonalmente (DAMASCENO et al., 2015; SOUSA et al., 2019; ABREU et al., 2020).

Neste contexto, a melhor explicação possível é que o SES pode estar completamente obsoleto e com progressiva deterioração de seus equipamentos. Isto permite o frequente contato e mistura entre as águas pluviais e esgotos sanitários mal condicionados (SOUSA et al., 2019; ABREU, et al., 2020). Estes fatores contribuem com o aumento de NOCD, invertendo a lógica de sua utilidade. Além disso, o NOCD do 1으 Semestre de 2017, NOCD $\left(\mathrm{Y}_{1}\right)$ variou significativamente com lab e Salubridade da categoria moderada (Salubm), com a Presença/Ausência da Rede de Esgoto (RE), além da densidade populacional (Dens). Aliás, este último parâmetro resultou significativo somente neste período, onde a variação de densidade de habitantes/bairro impactou NOCD $\left(Y_{1}\right)\left(R^{2}{ }_{A j}=0,56, p<0,0001\right)$.

É evidente que as zonas mais "quentes" das figuras 2 e 3 não se concentram apenas no centro sobre a mancha identificada da existência de RE, mas também parece ter alguma correlação com as zonas de drenagem (igarapés e canais) (SOUSA et al., 2019). A esse respeito é também possível inferir que o SES existente não é capaz de reduzir NOCD nos bairros de maior adensamento populacional ("zonas quentes"), provavelmente devido às baixas taxas de investimentos neste setor, mantendo uma precária e histórica ausência desses serviços, mas também ao avanço da malha urbana para locais com maior densidade de drenagem sob influência de marés (SOUSA et al., 2019; ABREU et al., 2020).

Nas figuras 2 e 3 é possível notar que NOCD é sempre maior nos períodos chuvosos (cores "quentes"), e justamente onde coexistem bairros com salubridade mediana (ISA mediano) e também onde há presença da RE ou SES (Figura 5). Como a elevada taxa de precipitação no 10 semestre (até $420 \mathrm{~mm} / \mathrm{mês}$ ) tende a expor a população a maior risco sanitário neste período (CUNHA et al., 2004; CUNHA et al., 2005), principalmente nos bairros próximos à rede de esgoto e sujeitos às variações de marés, com mais alagamentos e acúmulo superficial de água que se infiltra na rede de esgoto, há comprometimento de sua eficiência, elevando a vazão das águas parasitárias e a probabilidade de prováveis doenças parasitárias e virais (SOUSA et al., 2019; CUNHA et al., 2014).

Esses fatores, associados com medianos e baixos indicadores de ISA e RE nos bairros, tendem a contribuir com o cenário do atual SES de Macapá. E como é evidente nestes casos, um sistema deficitário não é capaz de separar esgoto doméstico de águas pluviais, refletindo negativamente na saúde pública (CASTRO et al., 2009; MONTEIRO et al., 2011; ALDERMAN et al., 2012). A existência de uma rede de esgoto não é garantia de qualidade e proteção à saúde. E equipamentos ou estruturas sanitárias obsoletas (lab, les, 
Idr e Irs) parecem ser potencialmente prejudiciais a NOCD.

Fatores como a existência de infiltração das águas próximos das redes de esgotos antigas, com transbordamento para as vias públicas próximos à rede de drenagem, tendem a contaminar as águas pluviais e, por consequência, os cidadãos que entram em contato com essas águas (KUMAR et al., 2016; VAN BIJNEN et al., 2018). Este é um problema frequente e os impactos e problemas de saúde são também recorrentes (AHMED et al., 2019), resultando em surtos de diarreia no entorno destes locais (MARA et al., 2010).

As informações de NOCD nos mapas de calor possibilitam observar como os impactos das ações estruturantes e não estruturantes refletem nos indicadores de saúde pública. Contudo, a expansão urbana e a falta de ordenamento territorial também geram desigualdades socioeconômicas relevantes, as quais são muito prejudiciais ao planejamento e soluções técnicas do setor (RUBIO et al., 2017; LI et al., 2018; WANG et al., 2019). Entretanto, as zonas de NOCD mais 'frias' nos mapas, são justamente os bairros mais afastados ou isolados em termos urbanos. Isso quer dizer que, apesar da inexistência de RE (SES mais precários e considerados pouco salubres), são surpreendentemente os bairros onde há menores ocorrências de NODC.

Neste aspecto, a integração entre análise estatística de variáveis sanitárias e ambientais com a espacialização geográfica de NOCD (GIS) mostra-se como uma abordagem robusta para evidenciar e testar padrões de variação espaço-sazonal dos indicadores de saúde pública e sanitários (STATHAKIS et al., 2018; LANDIM, 2015). Assim, espera-se ter sido possível descrever o panorama atual do SES de Macapá, para uma interpretação da gestão sanitária e ambiental municipal. Tais evidências servem como referencial e base para outros estudos envolvendo estes indicadores sanitários e de saúde pública, os quais são de eminente interesse científico e socioambiental.

\section{CONCLUSÕES}

Os bairros medianamente salubres (ISA mediano), e atendidos pelo serviço de coleta de esgoto em Macapá (RE), são os que apresentaram maior frequência de NOCD durante 2017 e 2018, ocorrendo o inverso nos bairros menos salubres (ISA baixo). Assim, rejeita-se a hipótese principal da pesquisa.

Sazonalmente foi observado um padrão oscilatório de variação entre os casos de diarreia entre os períodos sazonais de 2017 e 2018. Contudo, como esperado, os períodos chuvosos foram os que apresentaram maiores NOCD. E essa variação pode ser explicada pela condição e/ou deficiência das estruturas sanitária e do ambiente de entorno, período sazonal. Mas, curiosamente, nenhum parâmetro da qualidade da água explica o NOCD. Entretanto, a RE apresentou correlação ou explicabilidade de até $50 \%$ de NOCD, sendo possível afirmar que o SES não opera com sua função protetora sanitária e de saúde pública. Pelo contrário, parece tender a piorar esse indicador. Espacialmente, as cores mais 'quentes' de NOCD ocorreram nas zonas urbanas mais 'estruturadas', próximas ao centro, principalmente no período chuvoso. Mas foi possível verificar que os bairros mais afastados (zonas mais 'frias') apresentaram menores NOCD, sugerindo um paradoxo espaço-sazonal entre NOCD e infraestrutura sanitária.

Há limitações na presente investigação, pois a defasagem temporal entre as séries históricas analisadas permite apenas uma aproximação da variação espacial, mas não da variação temporal de NOCD 
em relação às variáveis explicativas. Espera-se que esta pesquisa sirva como uma contribuição ao tema, considerando a profunda ausência de séries históricas nesta área na Amazônia e no Estado do Amapá.

\section{REFERÊNCIAS}

ABREU, C. H. M.; BARROS, M. L. C.; BRITO, D. C.; TEIXEIRA, M. R.; CUNHA, A. C.. Hydrodynamic modeling and simulation of water residence time in the estuary of the lower Amazon River. Water, v.12, n.3, p.660-689, 2020. DOI: http://dx.doi.org/10.3390/w12030660

AGUIAR, J. S.; SILVA, L. M. S.. Caracterização e avaliação das condições de vida das populações residentes nas ressacas urbanas dos municípios de Macapá e Santana. p.165-236. In: DIAGNÓSTICO DAS RESSACAS DO ESTADO DO AMAPÁ: BACIAS DO IGARAPÉ DA FORTALEZA E RIO CURIAÚ, MACAPÁ-AP, CPAQ/IEPA e DGEO/SEMA. Anais. 2003. p.165230.

AHMED, W.; HAMILTON, K.; TOZE, S.; COOK, S.; PAGE, D.. A review on microbial contaminants in stormwater runoff and outfalls: potential health risks and mitigation strategies. Science of The Total Environment, v.692, p.1304-1321, 2019. DOI:

http://dx.doi.org/10.1016/i.scitotenv.2019.07.055

ALDERMAN, K.; TURNER, L. R.; TONG, S.. Floods and human health: a systematic review. Environment International, v.47, p.37-47, 2012. DOI:

http://dx.doi.org/10.1016/j.envint.2012.06.003

ANNE-SOPHIE, M. H.; DORNER, S. M.; SAUVÉ, S.; ABOULFADL, K.; GALARNEAU, M.; SERVAIS, P.; PRÉVOST, M.. Temporal analysis of e. coli, tss and wastewater micropollutant loads from combined sewer overflows: Implications for Management. Environmental Science: Processes \& Impacts, v.17, n.5, p.965-974, 2015. DOI: http://dx.doi.org/10.1039/c5em00093a

ARAUJO, E. P.; CUNHA, H. F. A.; CUNHA, A. C.. Funcionamento operacional da estação de tratamento de água da CAESA (ETAM). In: SEMANA DE ENGENHARIA CIVIL DA UNIFAP, 4. Anais. Macapá, 2019. p.1-12.

BIDKHORI, M.; YOUSEFI, M.; ROHANI, H.; EBRAHIMI, H.; MOHAMMADI, A. A.. The influence of the use of improved sanitation facilities and improved drinking-water sources on the diarrhea-associated deaths in children under 5 years. Human And Ecological Risk Assessment: An International Journal, v.25, n.5, p.1234-1241, 2018. DOI: http://dx.doi.org/10.1080/10807039.2018.1462089

BRASIL. Ministério do Desenvolvimento Regional. 24을 Diagnóstico dos Serviços de Água e Esgotos. Brasília, 2019.

CASTRO, L.; BAPTISTA, M.; BARRAUD, S.. Sistemática de Validação de Indicadores para a Avaliação dos Efeitos da Urbanização nos Corpos de Água por meio de Análise Crítica. Revista Brasileira de Recursos Hídricos, v.14, n.4, p.103-112, 2009. DOI: http://dx.doi.org/10.21168/rbrh.v14n4.p103-112

CHAGAS, M. A.; SANTOS, J. S. A.; SILVA, E. L.. Política ambiental: a negligência do urbano na Amazônia. Pracs: Revista Eletrônica de Humanidades do Curso de Ciências Sociais da UNIFAP, v.9, n.1, p.127-136, 2016. DOI: http://dx.doi.org/10.18468/pracs.2016v9n1.p127-136

CRAWLEY, M. J.. The R Book. New Jersey: John Wiley \& Sons, LTDA, 2007.

CUNHA, A. C.; CUNHA, H. F. A.; BRASIL JÚNIOR, A. C. P.; DANIEL, L. A.; SCHULZ, H. E.. Qualidade microbiológica da água em rios de áreas urbanas e periurbanas no baixo Amazonas: o caso do Amapá. Engenharia Sanitária e Ambiental, v.9, n.4, p.322-328, 2004. DOI: http://dx.doi.org/10.1590/s1413-41522004000400009

CUNHA, A. C.; OLIVEIRA, B. S. G.. Correlação entre qualidade da água e variabilidade da precipitação no sul do Estado do Amapá. Ambiente e Água - An Interdisciplinary Journal of Applied Science, v.9, n.2, p.261-275, 2014. DOI: http://dx.doi.org/10.4136/ambi-agua.1287

CUNHA, A. C.; NAZARE, A. S.; PANTOJA, S.; CUNHA, H. F. A.; SOUZA, J.. Monitoramento de águas superficiais em rios estuarinos do estado do Amapá sob poluição microbiológica. In: Boletim do Museu Paraense Emílio Goeldi. Série Ciências Naturais, Belém-PA, v.1, n.1, p.191-199, 2005.

DAMASCENO, M. C. S.; RIBEIRO, H. M. C.; TAKIYAMA, L. R.; PAULA, M. T.. Avaliação sazonal da qualidade das águas superficiais do Rio Amazonas na orla da cidade de Macapá, Amapá, Brasil. Ambiente e Água: An Interdisciplinary Journal of Applied Science, v.10, n.3, p.598-613, 2015. DOI: http://dx.doi.org/10.4136/ambi-agua.1606

DECINA, T. G. T.; BRANDÃO, J. L. B.. Análise de desempenho de medidas estruturais e não estruturais de controle de inundações em uma bacia urbana. Engenharia Sanitária e Ambiental, v.21, n.1, p.207-217, 2016. DOI: http://dx.doi.org/10.1590/s1413-41520201600100116134

FILE, T.; DINKA, H.; GOLASSA, L.. A retrospective analysis on the transmission of Plasmodium falciparum and Plasmodium vivax: the case of Adama city, east shoa zone, Oromia, Ethiopia. Malaria Journal, v.18, n.1, p.1-5, 2019. DOI: http://dx.doi.org/10.1186/s12936-019-2827-6

GROTT, S. L.; FAÇANHA, E. B.; FURTADO, R. N.; CUNHA, H. F. A.; CUNHA, A. C.. Variação espaço-sazonal de parâmetros da qualidade da água subterrânea usada em consumo humano em Macapá, Amapá, Brasil. Engenharia Sanitária e Ambiental, v.23, n.4, p.645-654, 2018. DOI: http://dx.doi.org/10.1590/s1413-41522018162018

HELLER, L.; COLOSIMO, E. A.; ANTUNES, C. M. F.. Environmental sanitation conditions and health impact: a case-control study. Revista da Sociedade Brasileira de Medicina Tropical, v.36, n.1, p.41-50, 2003. DOI: http://dx.doi.org/10.1590/s0037-86822003000100007

IBGE. Instituto Brasileiro de Geografia e Estatística. Cidades e Estados: Macapá. IBGE, 2019.

IMADA, K. S.; ARAUJO, T. S.; MUNIZ, P. T.; PÁDUA, V. L.. 
Socioeconomic, hygienic, and sanitation factors in reducing diarrhea in the Amazon. Revista de Saúde Pública, v.50, p.111, 2016. DOI: http://dx.doi.org/10.1590/s1518$\underline{8787.2016050006505}$

INSTITUTO TRATA BRASIL. Ranking do Saneamento. São Paulo: Go Associados, 2020.

KUMAR, K.; KOZAK, J.; HUNDAL, L.; COX, A.; ZHANG, H.; GRANATO, T.. In-situ infiltration performance of different permeable pavements in a employee used parking lot: A four-year study. Journal of Environmental Management, v.167, p.8-14, 2016. DOI:

http://dx.doi.org/10.1016/j.jenvman.2015.11.019

LANDIM, P. M. B.. Sobre Geoestatística e mapas. Terrae

Didática, v.2, n.1, p.19-33, 2015. DOI:

http://dx.doi.org/10.20396/td.v2i1.8637463

LI, L.; LI, J.; JIANG, Z.; ZHAO, L.; ZHAO, P.. Methods of Population Spatialization Based on the Classification Information of Buildings from China's First National Geoinformation Survey in Urban Area: a case study of Wuchang District, Wuhan City, China. Sensors, v.18, n.8, p.122, 2018. DOI: http://dx.doi.org/10.3390/s18082558

MACAPÁ. Plano Municipal de Saneamento Básico de Macapá: Diagnóstico da Prestação dos Serviços de Saneamento Básico. Macapá: MPB Engenharia, 2016.

MARA, D.; LANE, J.; SCOTT, B.; TROUBA, D.. Sanitation and Health. Plos Medicine, v.7, n.11, p.1-7, 2010. DOI: http://dx.doi.org/10.1371/journal.pmed.1000363

MIAO, Z.; HAN, M.; HASHEMI, S.. The effect of successive low-impact development rainwater systems on peak flow reduction in residential areas of Shizhuang, China.

Environmental Earth Sciences, v.78, n.2, p.1-12, 2019. DOI: http://dx.doi.org/10.1007/s12665-018-8016-z

MONTEIRO, M. C.; PEREIRA, L. C. C.; GUIMARÃES, D. O. COSTA, R. M.; SOUZAFILHO, P. W. M.; VIEIRA, S. R.; JIMENEZ, J. A.. Influence of natural and anthropogenic conditions on the water quality of the Caeté river estuary (north Brazil). Journal of Coastal Research, v.64, p.1535-1539, 2011.

OLIVEIRA, B. S. S.; CUNHA, A. C.. Análise da qualidade da água como condição de contorno em estudos numéricos da hidrodinâmica e dispersão de poluentes químicos e microbiológicos em um trecho do Rio Jarí - AP. In: Ciências Exatas: Resultados dos projetos de Iniciação Científica da Univers. Fed. do Amapá (2009-2011). Macapá: UNIFAP, 2014. p.7-15.

OLIVEIRA, E. D. C.; CASTELO-BRANCO, R.; SILVA, L.; SILVA, N.; AZEVEDO, J.; VASCONCELOS, V.; FAUSTINO, S.; CUNHA, A.. First Detection of Microcystin-LR in the Amazon River at the Drinking Water Treatment Plant of the Municipality of Macapá, Brazil. Toxins, v.11, n.11, p.669-690, 2019. DOI: http://dx.doi.org/10.3390/toxins11110669

OMS. Organização Mundial de Saúde. Progress on drinking water, sanitation and hygiene. 2017.
OMS; UNICEF. Organização Mundial de Saúde; Fundo das Nações Unidas para a Infância. Joint Monitoring Programme for Water Supply, Sanitation and Hygiene. OMS, 2017

PEREIRA, J. P. F. N.; PEREIRA, N. N.; CUNHA, A. C.. Use of Automatic Identification System (AIS) data to study patterns of navigation behavior in the Macapá and Santana/AP/Brazil Ship Monitoring Area (AMN). Journal of Aquaculture \& Marine Biology, v.8, n.3, p.95-104, 2019. DOI: http://dx.doi.org/10.15406/jamb.2019.08.00247

R CORE TEAM. R: A language and environment for statistical computing. Vienna: R Foundation for Statistical Computing, 2018.

RUBIO, C.; RUBIO, M. C.; ABRAHAM, E.. Poverty Assessment in Degraded Rural Drylands in the Monte Desert, Argentina. An Evaluation Using GIS and Multi-criteria Decision Analysis. Social Indicators Research, v.137, n.2, p.579-603, 2017. DOI: http://dx.doi.org/10.1007/s11205-017-1606-4

SANTOS, L. F. P.. Indicadores de Salubridade Ambiental (ISA) e sua aplicação para a gestão urbana. Dissertação (Mestrado em Direito Ambiental e Políticas Públicas) Universidade Federal do Amapá, Macapá, 2012.

SNIS. Sistema Nacional de Informações sobre Saneamento. SNIS - Série Histórica. SNIS, 2019.

SOUSA, T. S.; VIEGAS, C. J. T.; CUNHA, H. F. A.; CUNHA, A. C.. Sistema de drenagem urbana no município de Macapá como dimensão relevante do saneamento básico. In: SEMANA DE ENGENHARIA CIVIL DA UNIFAP, 4. Anais. Macapá: UNIFAP, 2019. p.12.

STATHAKIS, D.; BALTAS, P.. Seasonal population estimates based on nighttime lights. Computers, environment and urban systems, v.68, 2018, p.133-141. DOI: http://dx.doi.org/10.1016/j.compenvurbsys.2017.12.001

TAVARES, J. P. N.. Características da climatologia de MacapáAP. Caminhos de Geografia, Uberlândia, v.15, n.50, p.138151, 2015.

VAN BIJNEN, M.; KORVING, H.; LANGEVELD, J.; CLEMENS, F.. Quantitative Impact Assessment of Sewer Condition on Health Risk. Water, v.10, n.3, p.245-263, 2018. DOI: http://dx.doi.org/10.3390/w10030245

VIEGAS, C. J. T.; SOUSA, T. S.; CUNHA, H. F. A.; CUNHA, A. C. A deficiência da rede de esgoto e seus impactos sobre o indicador de salubridade ambiental (Isa) de Macapá. In: SEMANA DE ENGENHARIA CIVIL DA UNIFAP, 4. Anais. Macapá: UNIFAP, 2019. p.1-12.

WANG, X.; LIU, G.; COSCIEME, L.; GIANNETTI, B. F.; HAO, Y.; ZHANG, Y.; BROWN, M. T.. Study on the emergy-based thermodynamic geography of the Jing-Jin-Ji region: combined multivariate statistical data with dmsp-ols nighttime lights data. Ecological Modelling, v.397, p.1-15, 2019. DOI: http://dx.doi.org/10.1016/j.ecolmodel.2019.01.021

A CBPC - Companhia Brasileira de Produção Científica (CNPJ: 11.221.422/0001-03) detém os direitos materiais desta publicação. Os direitos referem-se à publicação do trabalho em qualquer parte do mundo, incluindo os direitos às renovações, expansões e disseminações da contribuição, bem como outros direitos subsidiários. Todos os trabalhos publicados eletronicamente poderão posteriormente ser publicados em coletâneas impressas sob coordenação da Sustenere Publishing, da Companhia Brasileira de Produção Científica e seus parceiros autorizados. Os (as) autores (as) preservam os direitos autorais, mas não têm permissão para a publicação da contribuição em outro meio, impresso ou digital, em português ou em tradução. 